\title{
Programming hyperglycaemia in the rat through prenatal exposure to glucocorticoids - fetal effect or maternal influence?
}

\author{
M J Nyirenda, L A M Welberg and J R Seckl \\ Molecular Endocrinology Laboratory, Molecular Medicine Centre, University of Edinburgh, Western General Hospital, Edinburgh, UK \\ (Requests for offprints should be addressed to M J Nyirenda, Molecular Endocrinology Laboratory, Molecular Medicine Centre, University of Edinburgh, \\ Western General Hospital, Edinburgh EH4 2XU, UK; Email: m.nyirenda@ed.ac.uk)
}

\begin{abstract}
In a previous study, we showed that exposure of rats to dexamethasone (Dex) selectively in late pregnancy produces permanent induction of hepatic phosphoenolpyruvate carboxykinase (PEPCK) expression and hyperglycaemia in the adult offspring. The mechanisms by which glucocorticoids cause this programming are unclear but may involve direct actions on the fetus/neonate, or glucocorticoids may act indirectly by affecting maternal postnatal nursing behaviour. Using a cross-fostering paradigm, the present data demonstrate that switching the offspring at birth from Dex-treated dams to control dams does not prevent induction of PEPCK or hyperglycaemia. Similarly, offspring born to control dams but reared by Dex-treated dams from birth maintain normal glycaemic control. During the neonatal period, injection of saline per se was sufficient to cause exaggeration in adult offspring
\end{abstract}

responses to an oral glucose load, with no additional effect from Dex. However, postnatal treatment with either saline or Dex did not alter hepatic PEPCK activity. Prenatal Dex permanently raised basal plasma corticosterone levels, but under stress conditions there were no differences in circulating corticosterone levels. Likewise, Dex-exposed rats had similar plasma catecholamine concentrations to control animals. These findings show that glucocorticoids programme hyperglycaemia through mechanisms that operate on the fetus or directly on the neonate, rather than via effects that alter maternal postnatal behaviour during the suckling period. The hyperglycaemic response does not appear to result from abnormal sympathoadrenal activity or hypothalamic-pituitary-adrenal response during stress.

Journal of Endocrinology (2001) 170, 653-660

\section{Introduction}

Increasing epidemiological evidence in humans suggests that early life events are important determinants of common cardiovascular and metabolic diseases in adulthood (Barker et al. 1989, Hales et al. 1991, Curhan et al. 1996). These observations have been proposed to exemplify the phenomenon of 'programming', whereby a factor or manipulation acting in early life can alter the development of organs, causing permanent dysfunction and ultimately resulting in disease (Barker et al. 1993, Edwards et al. 1993). The mechanisms involved in this programming are unclear but the timing of exposure to an insult is thought to be particularly critical, perhaps because organs develop at different stages (and rates) and the susceptibility of a system to a programming agent will be highest during its critical developmental window(s).

Recently, we have shown that prenatal exposure to glucocorticoids in rats induces permanent hyperglycaemia in the adult offspring (Lindsay et al. 1996a, Nyirenda et al. 1998). This is associated with hyperinsulinaemia and a lifelong elevation in hepatic mRNA expression and activity of phosphoenolpyruvate carboxykinase (PEPCK)
(Nyirenda et al. 1998), a key enzyme of gluconeogenesis (Pilkis \& Granner 1992) - suggesting that an increase in hepatic glucose production contributes to the hyperglycaemia. Importantly, this programming occurred only when glucocorticoids were given during the last week of pregnancy and not with earlier treatment (Nyirenda et al. 1998). These findings indicate that the window of sensitivity to glucocorticoid programming of hyperglycaemia might lie late in gestation. In this model, a synthetic glucocorticoid, dexamethasone (Dex), is administered to the mother to avoid fetal compromise by repeated direct fetal injections. Thus, both the fetus and its mother received the glucocorticoid load, making it difficult to determine whether programming of hyperglycaemia is through direct action of glucocorticoids upon the fetus or indirectly through effects on the mother. For example, glucocorticoids are known to have profound effects on the central nervous system; they alter many aspects of cognition and behaviour (McLay et al. 1998, de Quervain et al. 1998) and are implicated in the pathogenesis of depression (Rubinow et al. 1984, Thakore \& Dinan 1995). Glucocorticoid exposure in late gestation might, therefore, influence maternal behaviour and postnatal care, thus 
indirectly affecting neonatal biology. This notion is supported by well established evidence demonstrating that changes in maternal postnatal behaviour can permanently influence offspring neuroendocrine function and behaviour (Meaney et al. 1989, Maccari et al. 1995). Thus, enrichment of the maternal postnatal environment programmes a favourable offspring hypothalamic-pituitaryadrenal (HPA) response, while maternal stress is associated with permanent HPA hyperactivity in the offspring (Meaney et al. 1989, Maccari et al. 1995). Prenatal Dex treatment mimics the effect of maternal postnatal stress, in that offspring of dams treated with Dex in late gestation have elevated basal corticosterone levels (Levitt et al. 1996). In humans, high adult plasma cortisol levels are found in individuals with low birth weight, and may contribute to the increased cardiovascular risk observed in these individuals (Levitt et al. 2000, Phillips et al. 2000). Low birth weight is also associated with high resting pulse rate in adult life (Phillips \& Barker 1997, Levitt et al. 2000), suggesting that the increased cardiovascular risk may, in part, be mediated by activation of the sympathoadrenal system. Indeed, patients with type 2 diabetes mellitus show increased sensitivity of blood pressure, glucose tolerance and vascular responsivity to catecholamines (Bruce et al. 1992, Hogikyan et al. 1999). These data suggest that altered development of mechanisms of the stress response may be important in mediating the effects of early environmental insult on later cardiovascular and metabolic control.

The aims of this study were (1) to determine whether hyperglycaemia programmed by prenatal glucocorticoid exposure is a result of fetal programming or is mediated indirectly by altering subsequent maternal postnatal behaviour; (2) to examine the effect of prenatal glucocorticoid treatment on the offspring stress response (HPA and sympathetic activity); (3) additionally, we examined whether a brief exposure to Dex just before birth or treatment in the postnatal period can reproduce the hyperglycaemic phenotype induced by treatment throughout the last week of pregnancy.

\section{Materials and Methods}

\section{Animals}

Wistar rats (200-250 g, Harlan UK Ltd, Bicester, Oxon, UK) were maintained under conditions of controlled lighting (lights on 0700-1900 h) and temperature $\left(22^{\circ} \mathrm{C}\right)$ and allowed free access to food (standard rat chow; $56.3 \%$ carbohydrate, $18 \cdot 3 \%$ protein, $\mathrm{NaCl} 0 \cdot 7 \%$; B.S.\&S Scotland Ltd, Edinburgh, UK) and tap water.

\section{Prenatal glucocorticoid exposure}

Time-mated female rats (10 per group) were given subcutaneous injections of either Dex $(100 \mu \mathrm{g} / \mathrm{kg} /$ day, dissolved in $4 \%$ ethanol- $0.9 \%$ saline, $200 \mu \mathrm{g} / \mathrm{ml}$ ) or vehicle during the last week of pregnancy (embryonic days (E) 15-21). Daily maternal weights were measured. In a separate cohort of rats, fasting plasma glucose and basal corticosterone levels were measured at day 20 of pregnancy. A cross-fostering experiment was undertaken at birth, with half the number of litters born to Dex-treated dams switched to and nursed by control dams, and vice versa. The offspring were weighed and litters were culled to eight; only litters born on the same day were crossed. There were no problems with pup acceptance by substitute mothers of either group. In further experiments to refine the window of Dex sensitivity, treatment with Dex or vehicle was restricted to the last two days of gestation (E20-21). To determine the effect of postnatal Dex exposure, pups born to untreated dams received subcutaneous injections of either Dex $(0.01 \mu \mathrm{g} / \mathrm{g})$ or saline on postnatal days 1,3 and 5 . Body weight was measured during and after treatment. Weaning was carried out at postnatal day 21 in all experiments, and adult male offspring, selected at random from each litter, were used in all subsequent experiments.

\section{Oral glucose tolerance test}

The offspring underwent an oral glucose tolerance test at the age of 6 months. Rats were handled regularly during the week before the experiment and habituated to being held in a folded tea towel. Animals were fasted overnight and tests were started between 0830 and $0900 \mathrm{~h}$ the following morning. Blood sampling was carried out by tail tipping from rats loosely restrained in the tea towel. A fasting sample was collected in heparinised eppendorf tubes at time 0 and further sampling was carried out 30 and $120 \mathrm{~min}$ after an oral glucose load of $2 \mathrm{~g} / \mathrm{kg}$ was given by gavage. Plasma was stored at $-20{ }^{\circ} \mathrm{C}$ or $-80{ }^{\circ} \mathrm{C}$ for glucose or catecholamine assay respectively. Glucose was determined by the enzymatic (hexokinase) method using a glucose assay kit supplied by Sigma Chemical Co. (Poole, Dorset, UK). The intra-assay and interassay coefficients of variation were $<2 \%$. Plasma catecholamine levels were estimated commercially at St Helier Hospital (Carshalton, Surrey, UK) using the high-pressure liquid chromatography (HPLC) method. The intra-assay coefficients of variation were $5 \%$ and $7 \%$ for noradrenaline and adrenaline respectively.

\section{Induction of stress by restraint procedure}

Basal corticosterone levels (6-month-old rats) were determined in tail tip blood samples collected at $0830 \mathrm{~h}$. To assess corticosterone response to stress, rats were placed in a Perspex cylinder $(7 \cdot 5 \mathrm{~cm}$ diameter $)$ for $20 \mathrm{~min}$ before returning them to their cages. Blood samples were 
Table 1 Maternal weight gain during the last week of pregnancy (3rd week), gestation length, littersize, male:female ratio and offspring birth weight in the three treatment groups. Results are means \pm S.E.M.

\begin{tabular}{|c|c|c|c|c|c|}
\hline & $\begin{array}{l}\text { Maternal weight } \\
\text { gain }(\mathrm{g})\end{array}$ & $\begin{array}{l}\text { Gestation length } \\
\text { (days) }\end{array}$ & Litter size & $\begin{array}{l}\text { Male:female } \\
\text { ratio }\end{array}$ & $\begin{array}{l}\text { Birth weight } \\
\text { (g) }\end{array}$ \\
\hline \multicolumn{6}{|l|}{ Treatment } \\
\hline Saline & $\begin{array}{l}81 \cdot 2 \pm 3 \cdot 3 \\
(n=10)\end{array}$ & $21 \cdot 1 \pm 0 \cdot 3$ & $10 \cdot 5 \pm 0 \cdot 6$ & $1 \cdot 3 \pm 0 \cdot 3$ & $\begin{array}{l}6 \cdot 2 \pm 0 \cdot 1 \\
(n=27)\end{array}$ \\
\hline Dex E20-21 & $\begin{array}{l}75 \cdot 8 \pm 4 \cdot 3 \\
(n=10)\end{array}$ & $21 \cdot 1 \pm 0 \cdot 5$ & $10 \cdot 6 \pm 0 \cdot 9$ & $0 \cdot 9 \pm 0 \cdot 3$ & $\begin{array}{l}6 \cdot 1 \pm 0 \cdot 2 \\
(n=31)\end{array}$ \\
\hline Dex E15-21 & $\begin{array}{l}58 \cdot 3 \pm 6 \cdot 7^{*} \\
(n=10)\end{array}$ & $21 \cdot 2 \pm 0 \cdot 4$ & $11 \cdot 4 \pm 1 \cdot 1$ & $1 \cdot 4 \pm 0 \cdot 4$ & $\begin{array}{l}5 \cdot 5 \pm 0 \cdot 1^{*} \\
(n=33)\end{array}$ \\
\hline
\end{tabular}

${ }^{*} P<0.05$ compared with saline treatment group.

collected $15 \mathrm{~min}$ into the restraint procedure and at further time points after the restraint. Plasma corticosterone levels were estimated as described below.

\section{Corticosterone assay}

Plasma samples were diluted in buffer $(135 \mathrm{mmol} / \mathrm{l}$ sodium borate, $0.5 \%$ bovine serum albumin, $1 \%$ methanol, $0 \cdot 1 \%$ ethylene glycol, $\mathrm{pH} 7 \cdot 4$ ) and heated at $70{ }^{\circ} \mathrm{C}$ to inactivate corticosterone-binding globulin. Antibody specific to corticosterone (final titre 1:10 000) was added along with $\left[{ }^{3} \mathrm{H}\right]$ corticosterone (Amersham International, Amersham, Bucks, UK), and samples were incubated at $4{ }^{\circ} \mathrm{C}$ overnight. Unbound activity was estimated in a beta counter (Minaxi Tricab 4000, Canberra Packard, Pangbourne, Bucks, UK) after addition of scintillant (Picofluoro 40, Canberra Packard). Corticosterone was estimated by comparison with unlabelled corticosterone standards (Sigma Chemical Co.). The intra-assay coefficient of variation was $4 \%$.

\section{PEPCK assay}

Fed animals were killed by decapitation between 0900 and $1000 \mathrm{~h}$ and the livers removed immediately. Fresh liver homogenates $(10 \%(\mathrm{w} / \mathrm{v}))$ were prepared at $0{ }^{\circ} \mathrm{C}$ in medium containing $250 \mathrm{mM}$ sucrose and $5 \mathrm{mM}$ Hepes, $\mathrm{pH} 7 \cdot 4$. Homogenates were centrifuged at $3600 \mathrm{~g}$ for $10 \mathrm{~min}$ to sediment intact cells. The supernatant fractions were further centrifuged at $130000 \mathbf{g}$ for $30 \mathrm{~min}$. The resulting supernatant (cytosol) was collected and stored at $-70{ }^{\circ} \mathrm{C}$ for PEPCK assay. Protein content of the preparations was determined by the method of Peterson (1977). PEPCK activity was assayed at $30^{\circ} \mathrm{C}$ by coupling the formation of oxalacetate with $\mathrm{NADH}$ oxidation in the presence of excess malate dehydrogenase (Boehringer Mannheim UK, Lewes, East Sussex, UK), as described by Petrescu et al. (1979). The reaction mixture (1 ml final volume) contained $50 \mathrm{mM}$ Hepes (pH 6.5), $50 \mathrm{mM}$ sodium bicarbonate, $1 \mathrm{mM} \mathrm{MnCl}, 0.25 \mathrm{mM} \mathrm{NADH}$, $1 \mathrm{mM}$ phosphoenolpyruvate, $1.5 \mathrm{U}$ malate dehydrogenase and $10 \mu \mathrm{l}$ of liver cytosolic preparation. The reaction was initiated with $0.15 \mathrm{mM}$ deoxy-GDP and a decrease in absorbance at $340 \mathrm{~nm}$ was followed for $5 \mathrm{~min}$. A reaction mixture lacking bicarbonate was used as control.

\section{Statistics}

All data are expressed as means \pm s.E.M. Data were compared using unpaired Student's $t$-tests or one-way ANOVA followed by Newman-Keuls post-hoc multiple comparisons test, where appropriate. Values were considered significant when $P<0 \cdot 05$.

\section{Results}

Gestational weight gain and offspring birth weight

Dex administration throughout the third week of gestation caused a significant reduction in maternal weight gain (Table 1). As can be predicted from the inhibitory effect on the HPA axis, Dex-treated dams had lower plasma corticosterone levels than controls $(3.7 \pm 0.7$ vs $16.5 \pm$ $2.5 \mu \mathrm{g} / \mathrm{dl})$. However, there were no significant differences in maternal plasma glucose concentrations between the groups $(6 \cdot 1 \pm 0.4$ vs $5 \cdot 5 \pm 0.3 \mathrm{mmol} / \mathrm{l})$. Likewise, the length of gestation, litter size or viability were not affected by Dex treatment (Table 1). As we have reported previously, administration of Dex throughout the third week of pregnancy caused a significant reduction in the birth weight of offspring (Nyirenda et al. 1998, and data included in Table 1). When treatment was restricted to the last two days of pregnancy, Dex neither altered maternal weight gain nor affected offspring birth weight (Table 1).

Prenatal Dex, cross-fostering at birth and offspring glucose homeostasis

Confirming our previous observations, the offspring of rats treated with Dex during the whole of the last week of pregnancy had permanent fasting and reactive hyperglycaemia, with elevated hepatic PEPCK activity (Nyirenda 

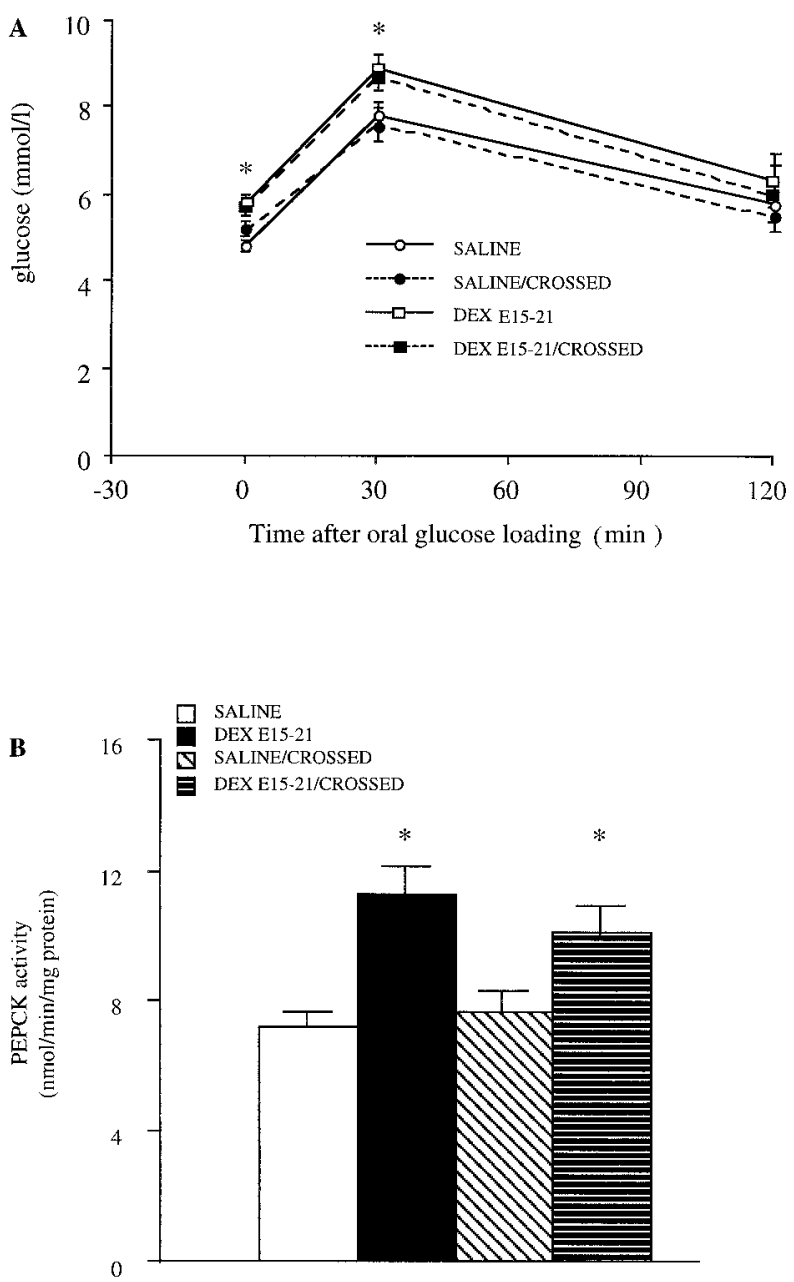

Figure 1 Cross-fostering and offspring glucose homeostasis. Pregnant rats received vehicle alone (SALINE) or Dex (DEX E15-21) during the third trimester. At birth, half the litters born to Dex-treated dams were cross-fostered and reared by saline-treated dams (DEX E15-21/CROSSED), while half the litters born to saline-treated dams were transferred to Dex-treated dams (SALINE/CROSSED). After an overnight fast, adult offspring underwent an oral glucose tolerance test (A) and hepatic PEPCK activity was measured from cytosolic preparations from fed rats (B). $n=9$ per group; ${ }^{*} P<0.05$ compared with SALINE and SALINE/CROSSED groups.

et al. 1998, and Fig. 1). We used this phenotype in a cross-fostering paradigm to establish whether the effect of Dex was on the fetus or was mediated postnatally through altered maternal nursing behaviour. Cross-fostering pups between control and Dex-treated dams did not change the pattern of glycaemic control in the adult offspring. Thus, offspring of Dex-treated dams had significantly higher levels of fasting glucose and showed exaggerated plasma glucose levels following an oral glucose challenge, irrespective of the treatment group of the rearing mother (Fig. 1A). Likewise, induction of hepatic PEPCK activity by
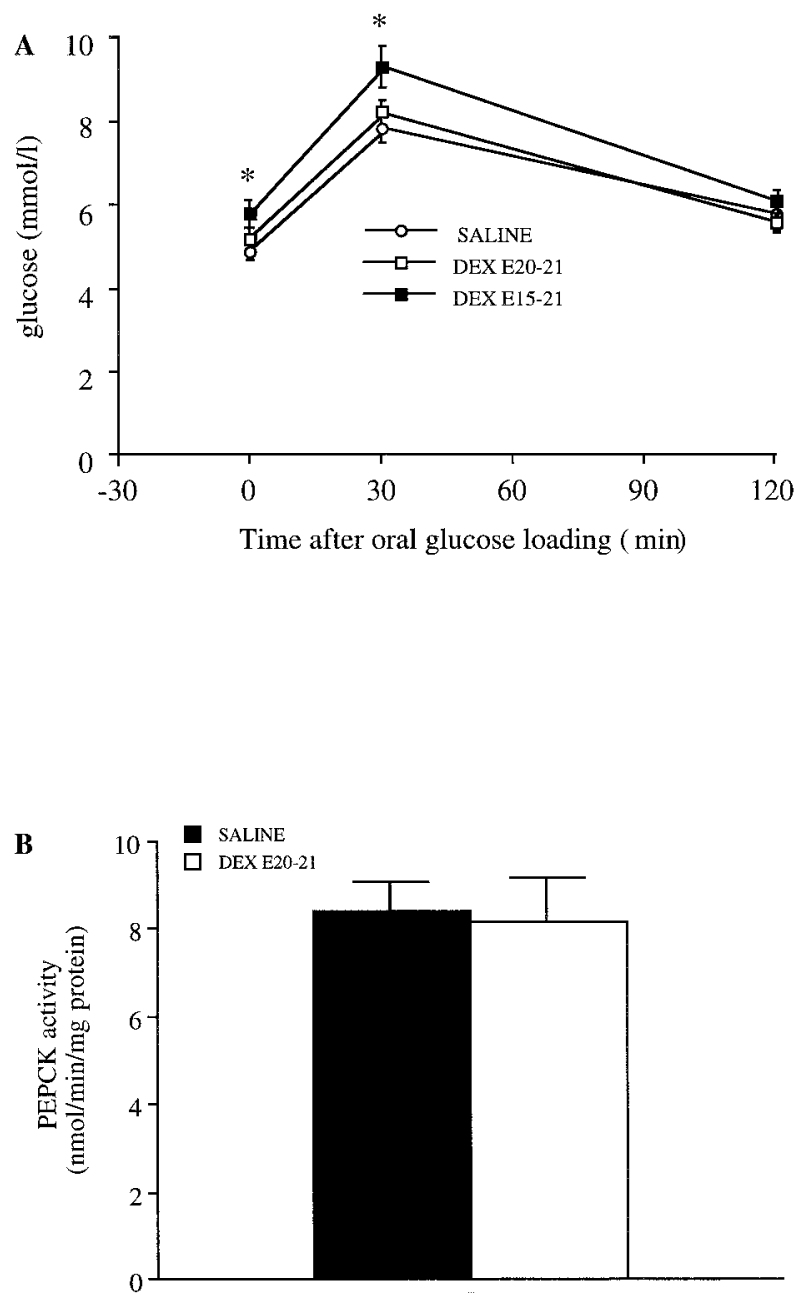

Figure 2 Length of prenatal Dex exposure and offspring glucose control. Pregnant rats were given Dex either throughout the third week of pregnancy (DEX E15-21, $n=8$ ) or during the last two days of gestation (DEX E20-21, $n=12$ ). A control group (SALINE, $n=8$ ) received vehicle throughout the third week of pregnancy. Following an overnight fast, adult offspring from each group underwent an oral glucose tolerance test (panel A). Panel B shows hepatic PEPCK activity in fed adult rats. ${ }^{*} P<0.05$ compared with SALINE group.

prenatal Dex administration was unaltered by crossfostering pups to control dams at birth (Fig. 1B). Offspring born to control dams exhibited normal glycaemic control and hepatic PEPCK activity, and these indices were not affected by cross-fostering of these offspring to Dex-treated dams (Fig. 1).

\section{Defining the window of Dex sensitivity}

Exposure to Dex on E20-21 and later glycaemic control When Dex was given to rats only during the last two days (instead of the entire last week) of pregnancy, 
Table 2 Effect of early postnatal Dex administration on subsequent body weight. Results are means \pm S.E.M.

\begin{tabular}{llll} 
& $\begin{array}{l}\text { Body weight } \\
\text { at } \mathbf{4} \text { weeks }(\mathrm{g})\end{array}$ & & $\begin{array}{l}\text { Body weight at } \\
\mathbf{6} \text { months }(\mathrm{g})\end{array}$ \\
\cline { 2 - 2 } $\begin{array}{lll}\text { Treatment } \\
\text { Postnatal saline }\end{array}$ & $\begin{array}{l}96 \cdot 4 \pm 10 \cdot 6(n=16) \\
\text { Postnatal Dex }\end{array}$ & & $492 \pm 24(n=16)$ \\
& $58 \cdot 9 \pm 8 \cdot 3^{*}(n=14)$ & & $478 \pm 18(n=14)$ \\
\hline
\end{tabular}

${ }^{*} P<0 \cdot 01$ compared with saline treatment group.

there was no significant effect on glycaemic control in the adult offspring (Fig. 2A). Likewise, hepatic PEPCK activity of offspring exposed to Dex during E20-21 was similar to the activity in control animals (Fig. 2B).

Postnatal Dex and glycaemic control in adulthood In order to test whether the window of programming of hyperglycaemia extended into the neonatal period, pups from untreated dams were given Dex or vehicle on postnatal days 1,3 and 5 . All pups survived after this treatment. Animals treated with Dex showed a slower rate of weight gain initially, so that at 4 weeks of age Dex-treated animals were $40 \%$ lighter compared with those that received saline injections (Table 2). However, by the age of six months the difference in body weight did not reach statistical significance (Table 2). There were no differences in fasting plasma glucose level, plasma glucose response to oral glucose load or hepatic PEPCK activity between animals that received saline or Dex postnatally (Fig. 3). Intriguingly, when compared with controls that were not manipulated postnatally, neonatal injection per se (either saline or Dex) induced an exaggerated glycaemic response to glucose challenge in the adult animals (uninjected $8 \cdot 0 \pm 0 \cdot 3$; postnatal saline $9 \cdot 4 \pm 0 \cdot 4 \mathrm{mmol} / \mathrm{l}$, $P<0 \cdot 05,30 \mathrm{~min}$ post loading). However, neonatal injection did not alter adult hepatic PEPCK activity (uninjected $7 \cdot 4 \pm 0 \cdot 5 ;$ postnatal saline $7 \cdot 7 \pm 0.6 \mathrm{nmol} / \mathrm{min} / \mathrm{mg}$ protein).

\section{The HPA and sympathoadrenal activity of the adult offspring}

In utero exposure to Dex has been shown to cause a lifelong elevation in basal (diurnal nadir, unstressed) corticosterone levels (Levitt et al. 1996) and increased hepatic glucocorticoid receptor expression (Nyirenda et al. 1998). An exaggerated stress response during the glucose tolerance procedure would result in increased glycaemic responses. Therefore, we examined whether prenatal exposure to Dex (DEX E15-21) was associated with an increase in corticosterone response to stress. As shown in Fig. 4, no significant difference was observed in plasma corticosterone responses to stress between animals that were exposed to Dex in utero and control animals. There was a tendency for higher concentrations of catecholamines in Dex-treated
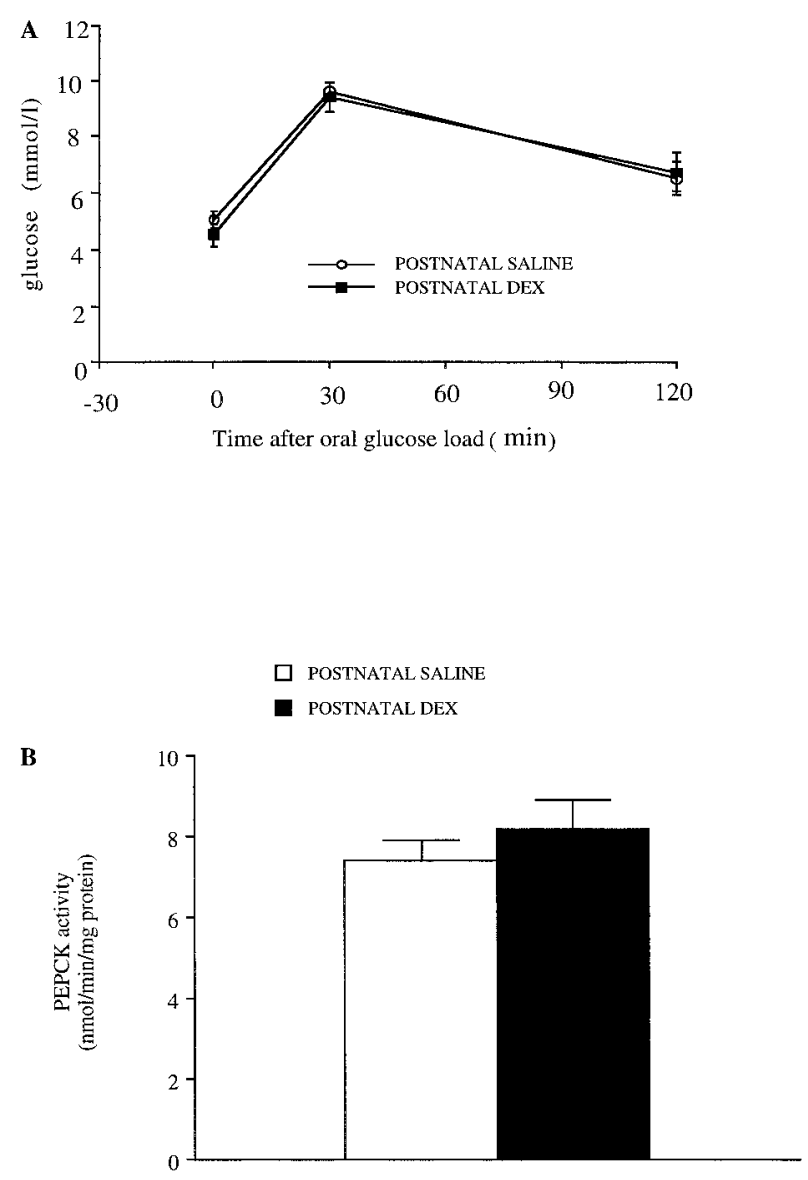

Figure 3 Effect of neonatal Dex on subsequent glucose homeostasis. Pups from untreated dams were given Dex (DEX, $n=14$ ) or saline (SALINE, $n=16$ ) in the first postnatal week. An oral glucose tolerance test was performed at 6 months of age following an overnight fast (A). Hepatic PEPCK activity was measured in cytosolic preparations from fed animals (B).

animals than in control rats, but this difference did not reach statistical significance (Table 3 ).

\section{Discussion}

Recent data have shown that exposure of pregnant rats and sheep to glucocorticoids, specifically Dex, produces permanent hyperglycaemia (Lindsay et al. 1996a, Nyirenda et al. 1998) and hypertension (Lindsay et al. 1996b, Dodic et al. 1998) in the adult offspring. Such programming of hyperglycaemia is associated with features of insulin resistance, as indicated by higher plasma insulinto-glucose ratio, and a permanent increase in hepatic PEPCK expression (Nyirenda et al. 1998). In the rat, both PEPCK activity and adult hyperglycaemia are induced selectively when glucocorticoids are given during the last week of pregnancy (Nyirenda et al. 1998). The 


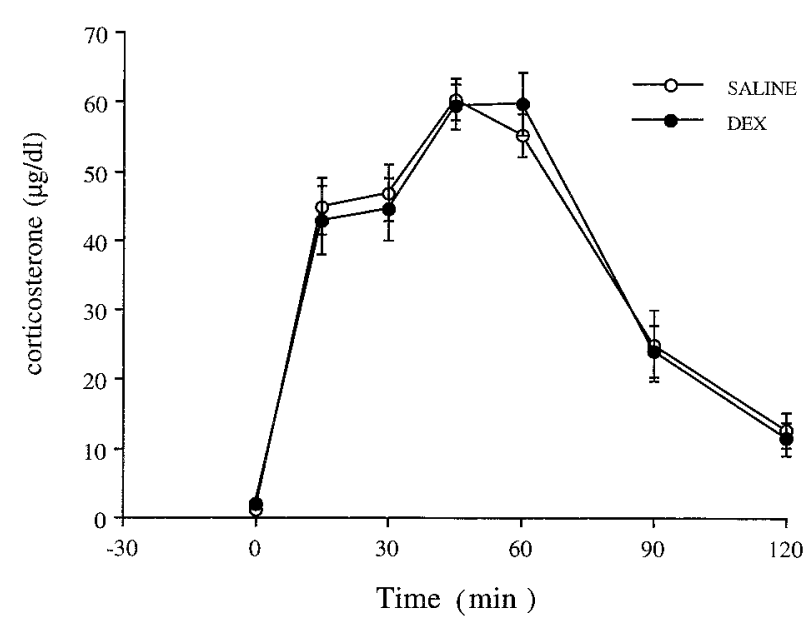

Figure 4 Plasma corticosterone response to stress. Basal plasma corticosterone concentrations were determined (time 0 ), and rats were then subjected to $20 \mathrm{~min}$ of restraint. Further samples were collected $15 \mathrm{~min}$ into, and at several time points after the restraint. Animals had been exposed to either vehicle alone (SALINE) or Dex (DEX E15-21) during the last week of intrauterine life.

mechanisms through which glucocorticoids mediate these effects are unclear. In particular, it remains unknown whether this programming reflects direct glucocorticoid action on late fetal development or results from their effects on the mother. It is well established that motherpup interactions, such as the level of maternal licking and grooming of pups, are crucial in programming essential biological systems such as responsiveness of the HPA axis (Meaney et al. 1989). Dex exposure in late gestation might, therefore, act on the mother to influence maternal behaviour and care during the suckling period and thus alter development of the offspring (Rubinow et al. 1984, Thakore \& Dinan 1995). Our data do not indicate a role for Dex-induced altered maternal nursing behaviour in glucocorticoid programming of hyperglycaemia; both induction of hepatic PEPCK activity and glycaemic control were determined by prenatal treatment, and were not affected by cross-fostering offspring between Dex-treated and control dams at birth. These findings strongly suggest that glucocorticoids programme PEPCK expression and hyperglycaemia by acting on the fetus in late gestation.

Evidence from human studies and other animal models also suggest that late gestation is a critical period in programming hyperglycaemia. In rats, diabetes (streptozotocin-induced) of the mother during pregnancy causes insulin resistance in the adult offspring, but normalisation of maternal hyperglycemia during the last week of gestation prevents insulin resistance in offspring (Aerts \& Van Assche 1992). However, frank maternal diabetes does not appear to be the underlying mechanism in programming hyperglycaemia by Dex, as this treatment did not significantly affect maternal plasma glucose levels in the present study. Epidemiological data have shown that, of people born during the Dutch famine, exposure to famine during the third trimester (but not earlier exposures) was associated with an increased risk of glucose intolerance and type 2 diabetes in adult life (Ravelli et al. 1998). How maternal malnutrition in late pregnancy predisposes the offspring to later abnormal glucose control remains unclear, but glucocorticoids provide a potential mechanism. For example, maternal nutritional restriction is associated with an increase in plasma levels of stress hormones (Fowden 1995) as well as attenuation of the placental enzyme barrier to glucocorticoids (LangleyEvans et al. 1996), thus increasing fetal glucocorticoid load.

The molecular details of glucocorticoid programming of hyperglycaemia remain to be elucidated but may involve metabolic pathways that develop in late gestation. Gluconeogenic enzymes provide one such potential target. These enzymes, amongst which PEPCK is rate-limiting (Pilkis \& Granner 1992), appear just before birth and are under tight glucocorticoid control (Ballard \& Hanson 1967, Friedman et al. 1993). Prenatal glucocorticoid treatment was associated with a permanent induction of hepatic PEPCK, supporting the role of altered gluconeogenesis in the programming of hyperglycaemia. Although PEPCK is induced at birth, restricting glucocorticoid treatment to the last two days of pregnancy (rather than treatment for the whole of the last week of pregnancy) did not cause a permanent induction in hepatic PEPCK and the adult offspring showed normal glycaemic control - suggesting that the window of sensitivity to glucocorticoids during late gestation is discrete. Prenatal exposure to glucocorticoids may also alter later glucose metabolism by influencing development of other target organs such as skeletal muscle, adipose tissue and pancreas, as well as mechanisms that co-ordinate the function of these organs. In particular, there is evidence that the adult HPA axis and the sympathoadrenal activity may be increased by

Table 3 Plasma catecholamine concentration during oral glucose tolerance testing in the saline- or Dex-treated groups. Results are means \pm S.E.M.

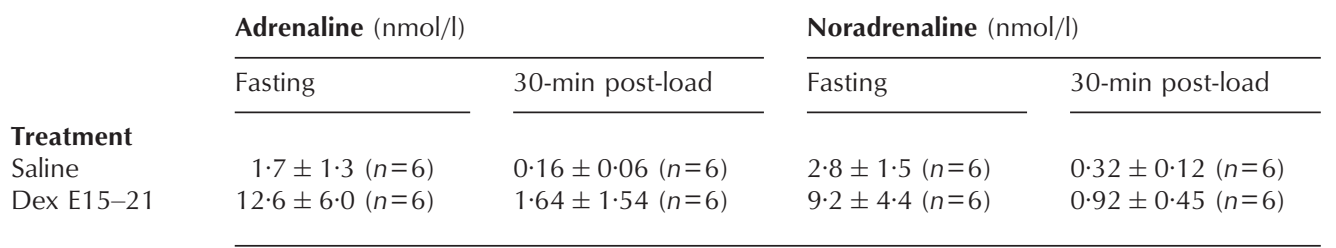


environmental stress in early life (Levitt et al. 2000, Phillips et al. 2000). It is thus intriguing that prenatal Dex exposure in rats caused lifelong elevation in basal corticosterone levels (Levitt et al. 1996) and hepatic glucocorticoid receptor expression (Nyirenda et al. 1998), although these animals had a similar plasma corticosterone response to stress as controls. Whatever the mechanism, our data do not suggest that increased glucose and PEPCK responses are a result of exaggerated corticosterone or catecholamine responses to stress, although increased basal glucocorticoid levels may be more pertinent.

In most mammalian species organ development continues well into the early postnatal period, making this another potential programming window. Indeed, there are several examples showing that manipulations during the neonatal period can induce permanent physiological and behavioural alterations (Arai \& Gorski 1968, Gustafsson \& Sternberg 1974, Csaba et al. 1979, Meaney et al. 1989). Therefore, we examined whether direct administration of Dex to the newborn would reproduce the phenotype of adult hyperglycaemia and elevated hepatic PEPCK expression. Intriguingly, pups that had saline injections showed higher adult glycaemic responses compared with animals that were untouched neonatally. This suggests that the stress of neonatal injection per se (perhaps through increased endogenous glucocorticoid levels) is enough to induce perturbation in later glycaemic control. Additional glucocorticoid loading with Dex postnatally had no further effect to injection alone, suggesting that either this mechanism is 'saturated' by the corticosterone stress response at this age (although this is usually slight during the immediate postnatal 'stress-hyporesponsive' period (Sapolsky \& Meaney 1986)) or, perhaps more plausibly, that other components of the stress response are involved. In this light, the role of perinatal manipulations in determining the sensitivity of the pancreas and liver to catecholamines and their effects upon glucose-insulin homeostasis has received some attention (Huff et al. 1991). Unlike the effect of prenatal Dex, the hyperglycaemic responses that followed neonatal injections were not associated with an induction of adult hepatic PEPCK expression, again suggesting that different mechanisms may be involved.

Glucocorticoids are widely used in obstetric practice, as immunosuppressants for maternal disorders (Rayburn 1992) and for short term treatment of the fetus to accelerate lung maturation in preterm labour (Crowley et al. 1990). Additionally, long term use of glucocorticoids has been advocated for prenatal therapy of fetuses at risk of congenital adrenal hyperplasia (Forest et al. 1989, Carlson et al. 1999). Experimental data suggests that exposure to glucocorticoids during development is associated with a risk of later metabolic and cardiovascular disorders. Rigorous follow-up studies are required to evaluate whether prenatal glucocorticoid exposure in humans produces these adverse effects. Indeed, recent data have suggested that a short antenatal glucocorticoid exposure in preterm babies is associated with increased blood pressure at 14 years (Doyle et al. 2000). Understanding of the action of glucocorticoids in development, including determination of the critical time window(s), may provide further insights into the pathogenesis of common cardiovascular and metabolic disorders, as well as allow a more rational therapeutic exploitation of glucocorticoids in early life.

\section{Acknowledgements}

These studies were supported by a Wellcome Senior Clinical Research Fellowship (J R S) and a Programme Grant from the Wellcome Trust (JR S). MJ N is the recipient of Overseas Research Studentship, a World Health Organisation fellowship and a Beit fellowship.

\section{References}

Aerts L \& Van Assche FA 1992 Islet transplantation in diabetic pregnant rats normalizes glucose homeostasis in their offspring. Journal of Developmental Physiology 17 283-287.

Arai Y \& Gorski RA 1968 Critical exposure time for androgenization of the developing hypothalamus in the female rat. Endocrinology $\mathbf{8 2}$ 1010-1014.

Ballard FJ \& Hanson RW 1967 Phosphoenolpyruvate carboxykinase and pyruvate carboxylase in the developing rat liver. Biochemical Journal 104 866-871.

Barker DJP, Osmond C, Goldings J, Kuh D \& Wadsworth MEJ 1989 Growth in utero, blood pressure in childhood and adult life, and mortality from cardiovascular disease. British Medical Journal 298 564-567.

Barker DJP, Gluckman PD, Godfrey KM, Harding JE, Owens JA \& Robinson JS 1993 Fetal nutrition and cardiovascular disease in adult life. Lancet 341 938-941.

Bruce DG, Chisholm DJ, Storlien LH, Kraegen EW \& Smythe GA 1992 The effect of sympathetic nervous system activation and psychological stress on glucose metabolism and blood pressure in subjects with type 2 (non-insulin-dependent) diabetes mellitus. Diabetologia 35 835-843.

Carlson AD, Obeid JS, Kanellopoulou N, Wilson RC \& New MI 1999 Congenital adrenal hyperplasia: update on prenatal diagnosis and treatment. Journal of Steroid Biochemistry and Molecular Biology 69 19-29.

Crowley P, Chalmers I \& Keirse M 1990 The effects of corticosteroid administration before preterm delivery: an overview of the evidence from controlled trials. British Journal of Obstetrics and Gynaecology 97 $11-25$.

Csaba G, Dobozy O, Lazary G \& Kaizer G 1979 Possibility of longlasting amplification of insulin receptors by a single treatment at new born age. Physiologica Academiae Scientiarum Hungaricae 53 487-492.

Curhan GC, Willett WC, Rimm EB, Spiegelman D, Ascherio AL \& Stampfer MJ 1996 Birth weight and adult hypertension, diabetes mellitus, and obesity in US men. Circulation 94 3246-3250.

Dodic M, May C, Wintour E \& Coghlan J 1998 An early prenatal exposure to excess glucocorticoid leads to hypertensive offspring in sheep. Clinical Science 94 149-155.

Doyle LW, Ford GW, Davis NM \& Callanan C 2000 Antenatal corticosteroid therapy and blood pressure at 14 years of age in preterm children. Clinical Science 98 137-142.

Edwards CRW, Benediktsson R, Lindsay R \& Seckl JR 1993 Dysfunction of the placental glucocorticoid barrier: a link between the foetal environment and adult hypertension? Lancet 341 355-357. 
Forest MG, Betuel H \& David M 1989 Prenatal treatment in congenital adrenal hyperplasia due to 21-hydroxylase deficiency: update 88 of the French multicentric study. Endocrine Research 15 277-301.

Fowden AL 1995 Endocrine regulation of fetal growth. Reproduction, Fertility and Development 7 351-363.

Friedman JE, Yun JS, Patel YM, McGrane MM \& Hanson RW 1993 Glucocorticoids regulate the induction of phosphoenolpyruvate carboxykinase (GTP) gene expression during diabetes. Journal of Biological Chemistry 268 12952-12957.

Gustafsson JA \& Sternberg A 1974 Irreversible androgenic programming at birth of microsomal and soluble rat liver enzymes active on 4-androstene-3,17-dione and 5-alpha, androstene-3-alpha, 17-beta-diol. Journal of Biological Chemistry 249 711-718.

Hales CN, Barker DJP, Clark PMS, Cox LJ, Fall C, Osmond C \& Winter PD 1991 Fetal and infant growth and impaired glucose tolerance at age 64. British Medical Journal 303 1019-1022.

Hogikyan RV, Galecki AT, Halter JB \& Supiano MA 1999 Heightened norepinephrine-mediated vasoconstriction in type 2 diabetics. Metabolism 48 1536-1541.

Huff RA, Seidler FJ \& Slotkin TA 1991 Glucocorticoids regulate the ontogenic transition of adrenergic receptor subtypes in rat liver. Life Sciences 48 1059-1065.

Langley-Evans SC, Philips G, Benediktsson R, Gardner D, Edwards CRW, Jackson AA \& Seckl JR 1996 Maternal dietary protein restriction, placental glucocorticoid metabolism and the programming of hypertension. Placenta 17 169-172.

Levitt N, Lindsay RS, Holmes MC \& Seckl JR 1996 Dexamethasone in the last week of pregnancy attenuates hippocampal glucocorticoid receptor gene expression and elevates blood pressure in the adult offspring in the rat. Neuroendocrinology 64 412-418.

Levitt NS, Lambert EV, Woods D, Hales CN, Andrews R \& Seckl JR 2000 Impaired glucose tolerance and elevated blood pressure in low birth weight, non-obese, young South African adults: early programming of cortisol axis. Journal of Clinical Endocrinology and Metabolism 85 4611-4618.

Lindsay RS, Lindsay RM, Waddell BJ \& Seckl JR 1996a Prenatal glucocorticoid exposure leads to hyperglycaemia in the rat: studies with $11 \beta$-hydroxysteroid dehydrogenase inhibitor carbenoxolone. Diabetologia 39 1299-1305.

Lindsay RS, Lindsay RM, Edwards CRW \& Seckl JR 19966 Inhibition of $11 \beta$-hydroxysteroid dehydrogenase in pregnant rats and the programming of blood pressure in the offspring. Hypertension 27 1200-1204.

Maccari S, Piazza PV, Kabbaj M, Barbazanges A, Simon H \& Le Moal M 1995 Adoption reverses the long-term impairment in glucocorticoid feedback induced by prenatal stress. Journal of Neuroscience 15 110-116.

McLay R, Freeman S \& Zadina J 1998 Chronic corticosterone impairs memory performance in the Barnes maze. Physiology and Behaviour 63 933-937.
Meaney MJ, Aitken DH, Viau V, Sharma S \& Sarrieau A 1989 Neonatal handling alters adrenocortical negative feedback sensitivity and hippocampal type II glucocorticoid receptor binding in the rat. Neuroendocrinology 50 597-604.

Nyirenda MJ, Lindsay RS, Kenyon CJ, Burchell A \& Seckl JR 1998 Glucocorticoid exposure in late gestation permanently programs rat hepatic phosphoenolpyruvate carboxykinase and glucocorticoid receptor expression and causes glucose intolerance in adult offspring. Journal of Clinical Investigation 101 2174-2181.

Peterson GL 1977 A simplification of the protein assay method of Lowry et al. which is more generally applicable. Analytical Biochemistry 83 346-356.

Petrescu I, Bojan O, Saied M, Barzu O, Schmidt F \& Kuhnle FH 1979 Determination of phosphoenolpyruvate carboxykinase activity with deoxyguanine 5'-diphosphate as a nucleotide substrate. Analytical Biochemistry 96 279-281.

Phillips DI \& Barker DJ 1997 Association between low birthweight and high resting pulse in adult life: is the sympathetic nervous system involved in programming the insulin resistance syndrome? Diabetes Medicine 14 673-677.

Phillips DI, Walker BR, Reynolds RM, Flanagan DE, Wood PJ, Osmond C, Barker DJ \& Whorwood CB 2000 Low birth weight predicts elevated plasma cortisol concentrations in adults from 3 populations. Hypertension 35 1301-1306.

Pilkis SJ \& Granner DK 1992 Molecular physiology of regulation of hepatic gluconeogenesis and glycolysis. Annual Review of Physiology 54 885-909.

de Quervain D, Roozendaal B \& McGaugh J 1998 Stress and glucocorticoids impair retrieval of long-term spatial memory. Nature 394 787-790.

Ravelli AC, van der Meulen JH, Michels RP, Osmond C, Barker DJ, Hales CN \& Bleker OP 1998 Glucose tolerance in adults after prenatal exposure to famine. Lancet 351 173-177.

Rayburn W 1992 Glucocorticoid therapy for rheumatic diseases: maternal, fetal, and breast feeding considerations. American Journal of Reproductive Immunology 28 138-140.

Rubinow DR, Post RM, Savard R \& Gold PW 1984 Cortisol hypersecretion and cognitive impairment in depression. Archives of General Psychiatry 41 279-283.

Sapolsky RM \& Meaney MJ 1986 Maturation of the adrenocortical stress response - neuroendocrine control mechanisms and the stress hyporesponsive period. Brain Research Reviews 11 65-76.

Thakore JH \& Dinan TG 1995 Cortisol synthesis inhibition: a new treatment strategy for the clinical and endocrine manifestations of depression. Biological Psychiatry 37 364-368.

Received in final form 12 April 2001

Accepted 30 May 2001 